\title{
Nuclotron injection beam profiles measurement system
}

\author{
D. Egorov ${ }^{1}$, V. Elkin ${ }^{1}$, D. Donets ${ }^{1}$, D. Ponkin ${ }^{1}$ \\ ${ }^{1}$ Laboratory of High Energy Physics, Joint Institute for Nuclear Research, 6 Joliot-Curie, Dubna, \\ Moscow region, 141980, Russia
}

\begin{abstract}
The superconducting synchrotron Nuclotron [1] is the one of the main parts of the NICA/MPD project [2] in the Laboratory of High Energy Physics of JINR in Dubna. During the work on updating the Nuclotron control and monitoring systems the new injection beam profiles measurement system was developed, produced and successfully put into operation. The development process and operation results are described.
\end{abstract}

\section{Introduction}

The Nuclotron (Figure 1) is intended to accelerate nuclei and multicharged ions up to an energy of $6 \mathrm{GeV} / \mathrm{u}$ for the charge-to-mass ratio $\mathrm{q} / \mathrm{A}=0.5$. The accelerator magnetic ring with circumference of $251.1 \mathrm{~m}$ includes 96 dipole, 64 quadrupole, 32 correcting multipole SC-magnets. The maximum value of the magnetic field is about $2 \mathrm{~T}$.

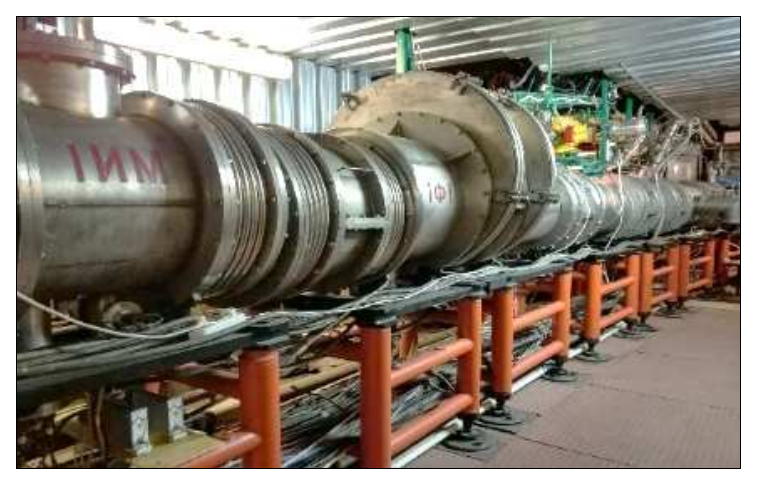

Figure 1. The Nuclotron ring

The injector (Alvaretz type linac LU-20) accelerates ions with a charge-to-mass ratio of $0.28<\mathrm{q} / \mathrm{A}<0.5$ up to $5 \mathrm{MeV} / \mathrm{u}$ and protons up to $20 \mathrm{MeV} / \mathrm{u}$. The injected beam transfer line is $30 \mathrm{~m}$ long and includes 2 bending magnets, 12 quadrupole 
lenses, and 12 correctors. The equipment of the single-turn injection comprises a superconducting septum magnet and kick electric plates.

The previous systems analog amplifiers low sensitivity and the long distance signal wires caused the upgrade necessity. PoE-based BPM power supply allows to put the BPM measurement module in close proximity to the profilometers.

The new injection beam profile measurement system allows to fine-tune the transport channel for more efficient beam injection into the Nuclotron ring.

\section{General description}

To analyze the beam transverse position 4 multi-wire beam monitors are used. The beam profile monitor consists of X- and Y-wire planes. Each plane which is assembled on a movable frame has 32 golden tungsten wires $0.1 \mathrm{~mm}$ in diameter separated by $2 \mathrm{~mm}$.

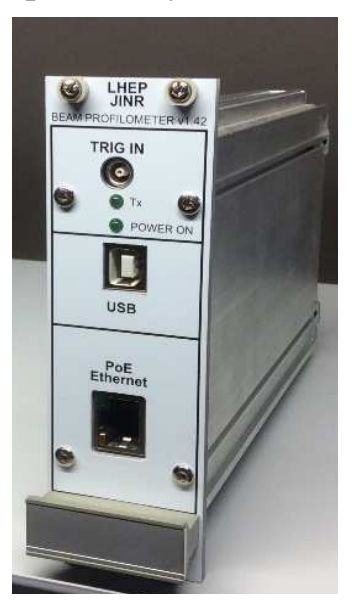

The measurements are provided by the BPM v1.42 designed at LHEP JINR (Figure 2). It is a multichannel, pulsed and DC low charge signals measurement module. The BPM $\vee 1.42$ specifications:

- Scale: $3 \mathrm{pC}, 6 \mathrm{pC}, 12 \mathrm{pC}$;

- Min Resolution: 0,001 pC;

- Number of channels: 42;

- Data Rate: up to $100 \mathrm{kSPS}$

- Minimum Integration time: $10 \mu \mathrm{s}$;

- Supply: PoE;

- ADC resolution: 14 bit;

- Modbus RTU over TCP interface;

- Case: EuroCard 3U 8HP.

Figure 2. The BPM v1.42

The BPM v1.42 contains a special charge-to-digital converter ICs. The ICs are 16-bit, 16-channel, current-input analog-to-digital converters (ADCs). The ADCs combines both current-to-voltage and analog-to-digital (A/D) conversion so that 16 separate low-level current output devices can be directly connected to its inputs and digitized.

For each of the 42 inputs, the BPM provides a dual-switched integrator frontend. This configuration allows for continuous current integration: while the on-chip $\mathrm{ADC}$ is digitizing one integrator, the other is integrating the input current. Adjustable integration times range is from $10 \mu$ s to $1 \mathrm{~ms}$.

A 32-bit ARM MCU using SPI bus (Serial Peripheral Interface) controls the ADCs. To configure ADCs full-scale range and measurement mode user must add a special bit sequence to the configuring command. The command word shall be transmitted to ADCs on global ADCs clock, not the SPI clock. This task was done using a state machine based on Xilinx XC9572 CPLD. 
Low noise and low temperature drift AD ADR444 $4.096 \mathrm{~V}$ voltage reference for the ADCs is used. The Ethernet communication is designed using Moxa MiiNePort E3 UART-to-Ethernet converter. The device is powered by MiiNePort E3 PoE interface and the special PoE controller IC-based cascade.

The BPM v1.42 has 42 data registers, 3 control registers and 1 status register. The registers can be read or written via Modbus RTU over TCP interface. As the device works in continuous or pulsed mode so the data reading process differs. The working mode is selected via the control register. In the continuous mode the input signal digitized at the moment of master request and the period could be from 50 ms up to seconds. In the pulsed mode case master should continuously analyze the status register. The input signal conversion is performed at the time of synchronization pulse occurs. At the data update moment, the status register value changes and master can read the new measurements result, the status register resets after the reading is complete.

The beam diagnostics software is integrated into the Tango controls-based acceleration complex control system [3, 4]. Tango-controls is a free open source device-oriented controls toolkit for controlling any kind of hardware or software and building SCADA systems [5]. It is an object oriented device control implemented in CORBA [6].

The software contains two parts: the Tango device servers and the Web-client (Figure 3).

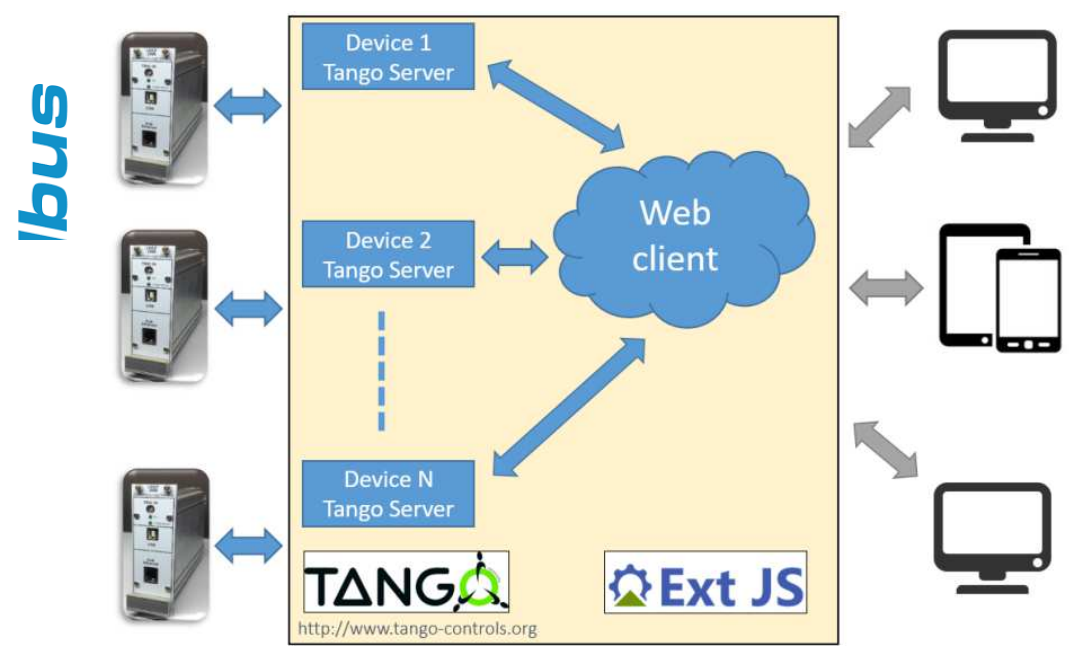

Figure 3. The injection beam profiles measurement system block diagram

The Tango device server module is used to communicate with hardware directly. The device server often has parameters such as read or write attributes, statuses, events and state information. The device server supports automatic retrieval of updated data, acquisition of data on demand, events generation and commands execution. Each BPM v1.42 device has own Tango device server. 
The Nuclotron injection beam profiles measurement systems device servers work in the Tango controls platform installed on the Debian OS running on the LHEP JINR virtual machine cluster.

By default, all the BPMs switched to a pulsed mode. The device servers read the new measurements result just after the synchronization signal occurs and the status register changes. After all the 42 data registers were read an update event occurs. The Web-client subscribed on the event is now reading the new data and performs plots update.

To monitor and manage the injection profiles measurements the Web-client was designed. The client contains a control panel and measured profiles plots area (Figure 4).

User can click the web page top area to open the panel with the devices settings. Each BPM has scale, integration time and zero level settings fields on this panel.

The light indicator in the upper panel fires at the data update moment. The indicator blinks every time an event occurs. It is also possible to save the reference profiles at the background for the channel tuning process.
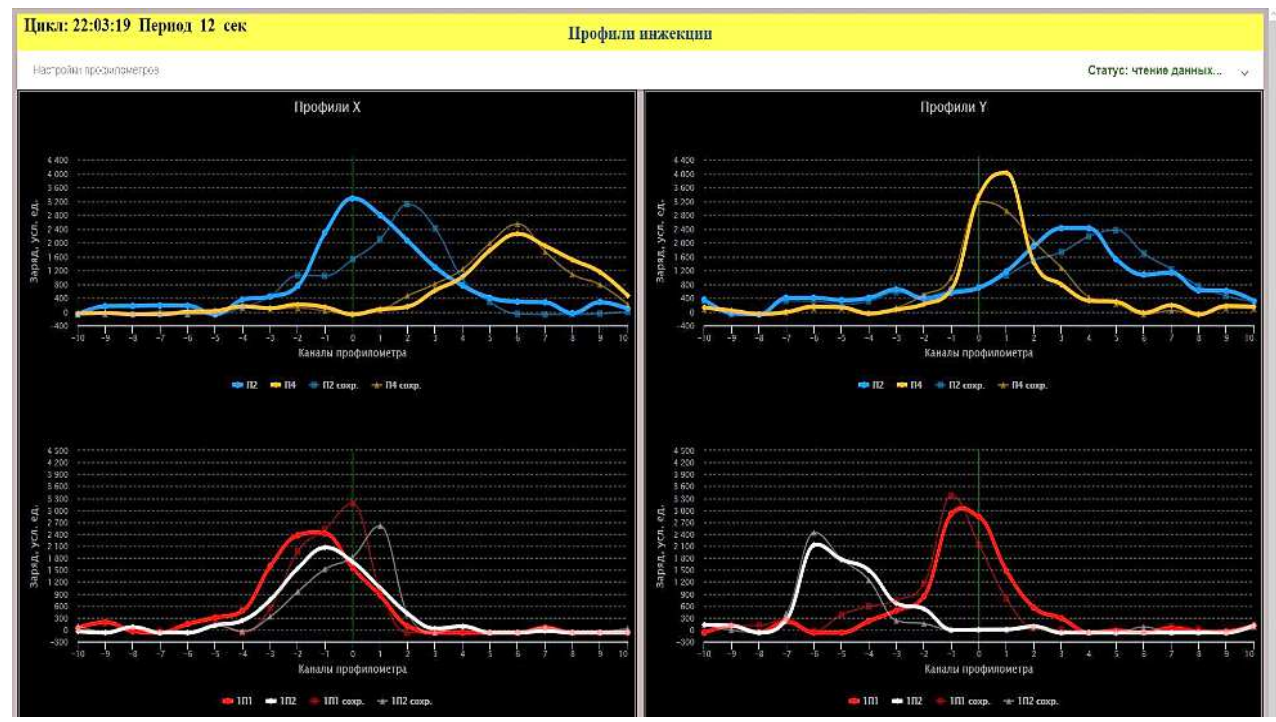

Figure 4. The client Web-page example

The client is based on the Angular platform [7]. Angular is a TypeScript-based open-source front-end web application platform. Execution of commands is allowed only from a certain IP. Authentication and authorization is done on the server through a special Tango controls module.

WebSocket device server module is used for the Tango device server and the Web-client data exchange. WebSocket [8] is a revolutionary new communication feature in the HTML5 specification, which defines a full-duplex communication channel that operates over the web through a single socket. This Tango controls module is used to connect Tango devices with the world using the WebSocket 
protocol. It supports automatic retrieval of updated data, acquisition of data on demand and commands execution. Compared to other protocols, such as HTTP (REST), the events subscribe ability allows a significant reduction in network traffic.

\section{Conclusion}

The new injection beam profiles measurement system was developed, produced and successfully put into operation. The Tango controls based distributed software was also designed to control and monitor injection beam profiles. The software was modified according to requests from users after first test run and currently works without any errors and bugs.

During the $55^{\text {th }}$ Nuclotron run the new injection beam profiles measurement system was used to fine-tune the transport channel for more efficient beam injection into the Nuclotron ring.

\section{References}

1. A. Sidorin, N. Agapov, V. Alexandrov, O. Brovko, G. Trubnikov etc. Status of the Nuclotron. «Nuclotron-M» project. Proceedings of IPAC'10, Kyoto, Japan. https://accelconf.web.cern.ch/accelconf/IPAC10/papers/mopd008.pdf

2. The NICA project official website http://nica.jinr.ru/

3. V. A. Andreev, V. I. Volkov, E. V. Gorbachev, V. A. Isadov, A. E. Kirichenko, S. V. Romanov, and G. S. Sedykh, TANGO Standard Software to Control the Nuclotron Beam Slow Extraction, Physics of particles and nuclei letters vol. 13 no. 5 pp. 951-956, (2016).

4. Evgeny V. Gorbachev, Vasiliy Andreev etc., Nuclotron and NICA control system development status, Proceedings of ICALEPCS2015, Melbourne, Australia - Pre-Press Release 23-Oct-2015 11:00MOPGF149.

5. The Tango controls official website. http://www.tango-controls.org/

6. J-M.Chaize, A.Gotz, “ W-D.Klotz, J.Meyer, M.Perez, E.Taurel, TANGO - an object oriented control system based on CORBA, Proceedings of ICALEPCS'99.

7. The Angular framework official website. https://angular.io/

8. The WebSocket protocol official website. http://websocket.org/index.html 